PROCEEDINGS OF THE

AMERICAN MATHEMATICAL SOCIETY

Volume 138, Number 10, October 2010, Pages 3723-3732

S 0002-9939(10)10364-5

Article electronically published on May 12, 2010

\title{
THE MINIMAL VOLUME ORIENTABLE HYPERBOLIC 2-CUSPED 3-MANIFOLDS
}

\author{
IAN AGOL \\ (Communicated by Daniel Ruberman)
}

\begin{abstract}
We prove that the Whitehead link complement and the $(-2,3,8)$ pretzel link complement are the minimal volume orientable hyperbolic 3 -manifolds with two cusps, with volume $3.66 \ldots=4 \times$ Catalan's constant. We use topological arguments to establish the existence of an essential surface which provides a lower bound on volume and strong constraints on the manifolds that realize that lower bound.
\end{abstract}

\section{INTRODUCTION}

Jorgensen and Thurston proved that the volumes of hyperbolic 3-manifolds are well-ordered. Moreover, if a volume is an $n$-fold limit point of smaller volumes (of order type $\omega^{n}$ ), then there is a corresponding hyperbolic manifold of finite volume with precisely $n$ orientable cusps. The smallest volume one-cusped orientable hyperbolic manifolds were identified by Cao and Meyerhoff [5], one of which is the figure-eight knot complement, with volume $2.0298 \ldots=2 V_{3}$, where $V_{3}=1.01494 \ldots$ is the volume of a regular ideal tetrahedron. Recently, Gabai, Meyerhoff and Milley have identified the smallest volume orientable hyperbolic 3-manifold to be the Fomenko-Matveev-Weeks manifold, with volume 0.9427... [11. Moreover, they identify the 10 one-cusped orientable manifolds with volume $<2.848$ (having five distinct volumes). It is an interesting question to find the smallest volume manifolds with $n$ orientable cusps. Adams showed that an $n$-cusped hyperbolic manifold has volume $\geq n V_{3}[1$. For orientable manifolds with two cusps, this was improved by Yoshida to a lower bound of 2.43 in 22 . In this paper, we prove that the Whitehead link complement $\mathbb{W}$ and the $(-2,3,8)$ pretzel link complement $\mathbb{W}^{\prime}$ are the minimal volume orientable hyperbolic 3-manifolds with two cusps, with volume $V_{8}=3.66 \ldots=4 \times$ Catalan's constant. These have been the smallest volume known 2 -cusped orientable hyperbolic 3-manifolds for quite some time, and we take it as given that these are known to have this volume (see [21] or [20, p. 474]). In fact, in Theorem 3.5, we prove that if $M$ is a hyperbolic manifold with a cusp and an essential surface disjoint from the cusp, then $\operatorname{Vol}(M) \geq V_{8}$, from which the result for 2-cusped manifolds follows in Theorem 3.6 by a result of Culler and Shalen [7].

Received by the editors July 9, 2008 and, in revised form, January 5, 2010.

2010 Mathematics Subject Classification. Primary 57M50.

The author was partially supported by NSF grant DMS-0504975 and the Guggenheim Foundation.

(C)2010 American Mathematical Society

Reverts to public domain 28 years from publication 
We now briefly outline the argument. Let $M$ be a compact orientable manifold with at least one boundary component, whose interior $\operatorname{int}(M)$ admits a finite volume hyperbolic metric and which contains a closed essential surface $X$ disjoint from the boundary. If the complement of $X$ has trivial "guts" (that is, the double $D(M \backslash \backslash X)$ of $M$ split along $X$ is a graph manifold), then we use the JSJ decomposition of the complement of $X$ to show that the boundary may be used to "cut up" the surface $X$, repeating until we get a surface which has nontrivial guts. Essentially what we are doing is replacing the initial surface $X$, which may have accidental parabolics, with a surface which has no accidental parabolics, but we must use a topological approach rather than geometric in order to keep track of the JSJ decomposition and get an acylindrical part of the guts in the end. We then apply a volume estimate from [2] and volume estimates of Miyamoto [13] giving lower bounds on volumes of hyperbolic manifolds with totally geodesic boundary to get the volume lower bound on $\operatorname{int}(M)$ (Theorem 3.5). A recent sharp estimate [3] implies that we may characterize completely the case of equality and identify the two smallest volume manifolds with two cusps by a simple combinatorial analysis (Theorem 3.6). The arguments of [2, 3] depend strongly on Perelman's proof of the geometrization conjecture (see [19, 18, 12, 14, 6, 15).

\section{Definitions}

In this section, we set up some notation and terminology for the theory of characteristic submanifolds, which is a relative version of the geometric decomposition.

For a surface $X \subset M$, we will use $M \backslash \backslash X$ to indicate the path-metric closure of $M \backslash X$. Let $M$ be an irreducible, orientable manifold. We will say that a properly embedded surface is essential if it is $\pi_{1}$-injective and $\partial \pi_{1}$-injective. Let $(X, \partial X) \subset$ $(M, \partial M)$ be a properly embedded essential surface. A compressing annulus for $X$ is an embedding

$$
i:\left(S^{1} \times I, S^{1} \times\{0\}, S^{1} \times\{1\}\right) \hookrightarrow(M, X, \partial M)
$$

such that

- $i_{*}$ is an injection on $\pi_{1}$,

- $i\left(S^{1} \times I\right) \cap X=i\left(S^{1} \times\{0\}\right)$, and

- $i\left(S^{1} \times\{0\}\right)$ is not isotopic in $X$ to $\partial X$.

An annular compression of $(X, \partial X) \subset(M, \partial M)$ is a surgery along a compressing annulus $i:\left(S^{1} \times I, S^{1} \times\{0\}, S^{1} \times\{1\}\right) \hookrightarrow(M, X, \partial M)$. Let $U$ be a regular neighborhood of $i\left(S^{1} \times I\right)$ in $M \backslash \backslash X$, let $\partial_{1} U$ be the frontier of $U$ in $M \backslash \backslash X$, and let $\partial_{0} U=\partial U \cap(X \cup \partial M)$. Then let $X^{\prime}=\left(X-\partial_{0} U\right) \cup \partial_{1} U$. The surface $X^{\prime}$ is the annular compression of $X$ (see Figure 11). We remark that if $X$ is essential, then $X^{\prime}$ is as well.

The notion of a pared manifold was defined by Thurston to give a topological characterization of geometrically finite hyperbolic 3-manifolds (see [16] or [4, Ch. 5]).

Definition 2.1. A pared manifold is a pair $(M, P)$, where

- $M$ is a compact, orientable irreducible 3 -manifold and

- $P \subset \partial M$ is a union of essential annuli and tori in $M$,

such that 

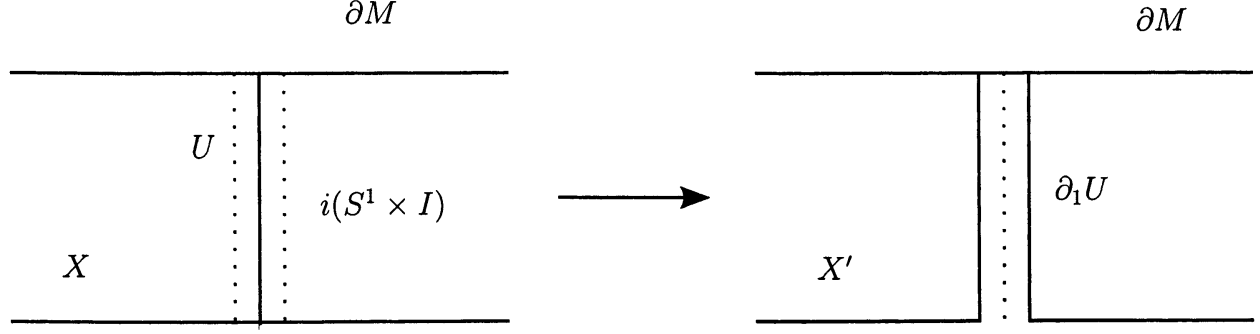

FiguRe 1. A annular compression of a surface (cross the picture with $\left.S^{1}\right)$.

- every abelian, noncyclic subgroup of $\pi_{1}(M)$ is peripheral with respect to $P$ (i.e., conjugate to a subgroup of the fundamental group of a component of $P)$ and

- every map $\varphi:\left(S^{1} \times I, S^{1} \times \partial I\right) \rightarrow(M, P)$ that is injective on the fundamental groups deforms, as maps of pairs, into $P$.

$P$ is called the parabolic locus of the pared manifold $(M, P)$. We denote by $\partial_{0} M$ the surface $\partial M-\operatorname{int}(P)$.

The motivation for the introduction of pared manifolds is the following geometrization theorem of Thurston:

Theorem 2.2 (4, Ch. 7]). If $(M, P)$ is an oriented pared 3-manifold with nonempty boundary, then there exists a geometrically finite uniformization of $(M, P)$.

The term uniformization means that there is a hyperbolic manifold $N$ such that $N$ is homeomorphic to $\operatorname{int}(M)$, and whose parabolic subgroups correspond to $P$ (see [4, Ch. 7] for more details).

Let $(M, P)$ be a pared manifold such that $\partial_{0} M$ is incompressible. There is a canonical set of essential annuli $(A, \partial A) \subset\left(M, \partial_{0} M\right)$, called the characteristic annuli, such that $(P, \partial P) \subset(A, \partial A)$, and characterized (up to isotopy) by the property that they are the maximal collection of nonparallel essential annuli such that every other essential annulus $(B, \partial B) \subset\left(M, \partial_{0} M\right)$ may be relatively isotoped to an annulus $\left(B^{\prime}, \partial B^{\prime}\right) \subset\left(M, \partial_{0} M\right)$ so that $B^{\prime} \cap A=\emptyset$. Each complementary component $L \subset M \backslash \backslash A$ is one of the following types:

(1) $T^{2} \times I$, a neighborhood of a torus component of $P$,

(2) $\left(S^{1} \times D^{2}, S^{1} \times D^{2} \cap \partial_{0} M\right)$, a solid torus with annuli in the boundary,

(3) (I-bundles, $\partial I$-subbundles), where the $I$-bundles over the boundary are subsets of $A$, or

(4) all essential annuli in $\left(L, \partial_{0} M \cap L\right)$ are parallel in $L$ into $(L \cap A, \partial(L \cap A))$.

The union of components of type (3), denoted $\left(W, \partial_{0} W\right) \subset\left(M, \partial_{0} M\right)$, is called the window of $\left(M, \partial_{0} M\right)$. It is unique up to isotopy of pairs.

The pared submanifold $\left(M-W, \partial(M-W)-\partial_{0} M\right)$ is denoted $G u t s(M, P)$. Note that the parabolic locus of $\operatorname{Guts}(M, P)$ will consist of characteristic annuli. If $M$ is compact orientable and $\operatorname{int}(M)$ admits a metric of finite volume, and if $(X, \partial X) \subset$ $(M, \partial M)$ is an essential surface, then define $\operatorname{Guts}(X)=\operatorname{Guts}(M \backslash \backslash X, \partial M \backslash \backslash \partial X)$. The components of type (4) are acylindrical pared manifolds, which have a complete hyperbolic structure of finite volume with geodesic boundary [16. We will 
let $\operatorname{Vol}(\operatorname{Guts}(M, P))$ denote the volume of this hyperbolic metric. If $D(M, P)$ is obtained by taking two copies of $M$ and gluing them along the corresponding surfaces $\partial_{0} M$, then $\operatorname{Vol}(\operatorname{Guts}(M, P))=\frac{1}{2} \operatorname{Vol}(D(M, P))$, where $\operatorname{Vol}(D(M, P))$ is the simplicial volume of $D(M, P)$, i.e. the sum of the volumes of the hyperbolic pieces of the geometric decomposition. If $\operatorname{Vol}(\operatorname{Guts}(M, P))=0$, then $M$ is a "book of $I$-bundles", with "pages" consisting of $W$, and "spine" consisting of solid tori and $T^{2} \times I$ (see [4, Example 2.10.4] for more information).

Let $V_{8}=4 \cdot K=3.66 \ldots$, where $K$ is Catalan's constant

$$
K=1-1 / 9+1 / 25-1 / 49+\cdots+(-1)^{n} /(2 n+1)^{2}+\cdots .
$$

Then $V_{8}$ is also the volume of a regular ideal octahedron in $\mathbb{H}^{3}$.

\section{EsSEntial SURFACES}

In this section, we prove the existence of an essential surface with nontrivial guts under certain hypotheses (Theorem 3.4). In the terminology of Culler and Shalen, we find a surface which is not a fibroid [9], i.e. whose complement is not a book of $I$-bundles. We start with an essential surface missing one cusp. If this surface has no accidental parabolics, we are done. If it does, we perform annular compressions until we get a surface with no accidental parabolics and which is not a fibroid.

Lemma 3.1. Let $(M, P)$ be a pared manifold, with window $\left(W, \partial_{0} W\right) \subset\left(M, \partial_{0} M\right)$. Suppose that there is a component $(J, K) \subset\left(W, \partial_{0} W\right)$ which is an I-bundle such that $\chi(J)<0$, and such that $K \cap P \neq \emptyset$. Let $K_{0}$ be a component of $K \cap P$. Then the surface $\partial_{0} M \cup K_{0}$ is compressible in $M$.

Proof. Let $Q$ be a surface such that $I \rightarrow J \rightarrow Q$ is a fiber bundle. Then $I \rightarrow K \rightarrow$ $\partial Q$ is a bundle over the boundary. Let $q_{0} \subset \partial Q$ be the component of $\partial Q$ such that $K_{0}$ fibers over $q_{0}$. Let $(\alpha, \partial \alpha) \subset\left(Q, q_{0}\right)$ be an essential arc. Then there is a disk $D \subset J$ which fibers over $\alpha$. The disk $D$ is essential, which implies that $\partial_{0} M \cup K_{0}$ is compressible. To see this, note that if $D$ is nonseparating in $M$, then it is essential. So we may assume that $D$ separates $M$, and therefore $D$ separates $J$. But $J$ is $\pi_{1}$-injective in $M$, and since $\alpha$ is essential in $Q, D$ is essential in $J$. Thus, each component of $J-D$ is $\pi_{1}$-injective in $J$, and therefore in $M$, and neither component of $J-D$ is simply connected. Therefore neither component of $\overline{M-D}$ can be a ball, since it contains a $\pi_{1}$-injective submanifold that is not simply connected, which implies that $D$ is essential, and therefore that $\partial_{0} M \cup K_{0}$ is compressible.

Lemma 3.2. Let $M$ be an orientable compact manifold such that int $(M)$ is hyperbolic of finite volume and so that $P=\partial M$ is the pared locus of $M$. Let $X \subset M$ be an essential surface. Then $(M \backslash \backslash X, P \backslash \backslash \partial X)$ is a pared manifold.

Proof. Since each component $V$ of $M \backslash \backslash X$ is $\pi_{1}$-injective, there is a covering space $\tilde{M} \rightarrow M$ such that there is a lift $M \backslash \backslash X \rightarrow \tilde{M}$ which is a homotopy equivalence. Any abelian noncyclic subgroup of $\pi_{1}(V)$ must be peripheral in $M$ and therefore in $\tilde{M}$ and $M \backslash \backslash X$. Any map $\varphi:\left(S^{1} \times I, S^{1} \times \partial I\right) \rightarrow(M \backslash \backslash X, P \backslash \backslash \partial X)$ that is injective on the fundamental groups deforms, as maps of pairs, into $P \backslash \backslash X \subset \tilde{M}$, since components of $P$ correspond to cusps associated to a complete hyperbolic structure on $\operatorname{int}(\tilde{M})$ induced from the hyperbolic structure on $\operatorname{int}(M)$. Thus, we see that $(M \backslash \backslash X, P \backslash \backslash \partial X)$ satisfies the hypotheses of a pared manifold. 
Lemma 3.3. Let $M$ be an orientable compact manifold such that int $(M)$ is hyperbolic of finite volume, so that $P=\partial M$ is the pared locus of $M$. Let $X \subset M$ be an essential surface. If $X$ has a compressing annulus, let $X_{1}$ be the surface obtained by performing an annular compression along $X$. Then $X_{1}$ has a pared annulus coming from $\partial M$ in the boundary of one of its gut regions.

Proof. Let $A \subset M \backslash \backslash X$ be a compressing annulus, with $\partial A=a_{0} \cup a_{1}$, such that $a_{0} \subset \partial M$, and $a_{1} \subset X$ is a closed curve in $X$ which is not boundary parallel in $X$. By Lemma 3.2. $(M \backslash \backslash X, P \backslash \backslash \partial X)$ is a pared manifold. Compression along $A$ creates the essential surface $X_{1}$ (see Figure 1). There will be a new component of the pared locus of $M \backslash \backslash X_{1}$ which is an annulus with core $a_{0}$. This annulus must be in a component of Guts $\left(X_{1}\right)$, because otherwise $a_{0}$ would lie in the boundary of the core of the window $W \subset M \backslash \backslash X_{1}$. This implies that $X$ had a compression, since reversing the annular compression to obtain $X$ from $X_{1}$ corresponds to adding a boundary annulus to an $I$-bundle of Euler characteristic $<0$, creating a compression by Lemma 3.1 .

Theorem 3.4. Let $M$ be an orientable compact 3-manifold whose interior admits a hyperbolic metric of finite volume. Suppose that $\partial M$ contains a torus $T_{1}$ and that there is a 2-sided essential surface $X_{0} \subset M$ such that $\partial X_{0} \cap T_{1}=\emptyset$. Then there is an essential surface $X \subset M$ such that $\chi(G u t s(X))<0$.

Proof. Let $\partial M=T_{1} \cup \cdots \cup T_{n}$, where $T_{i}$ is a torus. Then $\partial X_{0} \subset T_{2} \cup \cdots \cup T_{n}$. Suppose that $\chi\left(\operatorname{Guts}\left(X_{0}\right)\right)=0$. Then $M \backslash \backslash X_{0}$ is a book of $I$-bundles. Now, we perform a maximal sequence of annular compressions along $\partial M$ to obtain a sequence of essential surfaces $X_{1}, X_{2}, \ldots, X_{k} \subset M$, where $X_{i+1}$ is obtained from $X_{i}$ by an annular compression. Since $X_{0}$ is disjoint from $T_{1}$, the component of Guts $\left(X_{0}\right)$ incident with $T_{1}$ must be of the form $T^{2} \times I$, and thus $X_{0}$ has an annular compression. The compressing annulus comes from a curve in $T^{2}$ which is parallel to the boundary of a characteristic annulus of $M \backslash \backslash X_{0}$, and which is parallel to a curve in $T_{1}$ via a compressing annulus. For $i>0, X_{i}$ will be a 2 -sided essential surface, with a component of the pared locus coming from $\partial M$ lying in the boundary of a gut region of $M \backslash \backslash X_{i}$, by Lemma 3.3. If $\chi\left(\operatorname{Guts}\left(X_{i}\right)\right)=0$ for $i>0$, then the components of $\operatorname{Guts}\left(X_{i}\right)$ must be solid tori or tori $\times I$. Since $M$ is simple, the intersection of a pared annulus in $\partial M$ with the boundary of a solid torus component of $G u t s\left(X_{i}\right)$ must be primitive, and thus there is an annular compression of $X_{i}$ (see Figure 2). Any region of $\operatorname{Guts}\left(X_{i}\right)$ of the form $T^{2} \times I$ will also give rise to an annular compression of $X_{i}$, as we observed for $X_{0}$. Since $\chi\left(X_{i+1}\right)=\chi\left(X_{i}\right)$ and $X_{i+1}$ has two more boundary components than $X_{i}$, the number of annular compressions is finite, and thus at some stage we arrive at $X_{k}$ such that $\chi\left(\operatorname{Guts}\left(X_{k}\right)\right)<0$. Let $X=X_{k}$.

Theorem 3.5. Let $M$ be an orientable compact 3-manifold whose interior admits a hyperbolic metric of finite volume. Suppose that $\partial M$ contains a torus $T_{1}$ and that there is a 2-sided essential surface $X_{0} \subset M$ such that $\partial X_{0} \cap T_{1}=\emptyset$. Then $\operatorname{Vol}(M) \geq V_{8}$. If $\operatorname{Vol}(M)=V_{8}$, then $M$ has a decomposition into a single rightangled octahedron.

Proof. From Theorem 3.4, there is an essential surface $X \subset M$ such that $\chi(\operatorname{Guts}(X)) \leq-1$. By [3, Theorem 5.5], we have $\operatorname{Vol}(M) \geq \operatorname{Vol}(\operatorname{Guts}(X))$ (see 


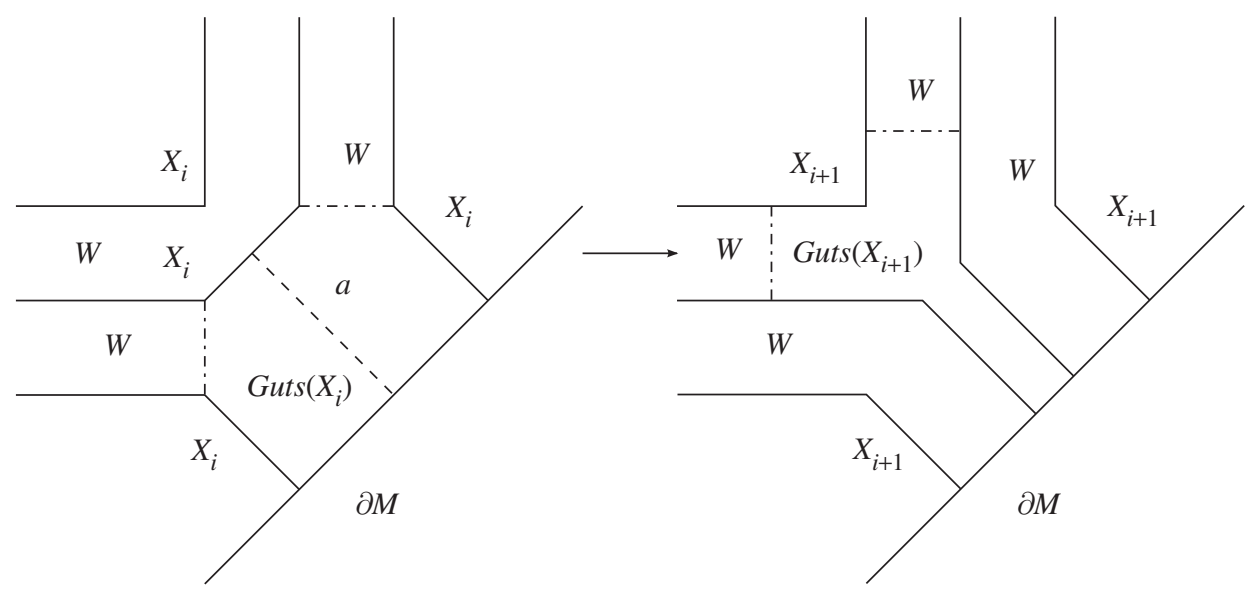

Figure 2. An annular compression $a$ of $X_{i}$ gives $X_{i+1}$ (cross the picture with $S^{1}$ ).

also [2, Theorem 9.1] on which the method of proof of [3] is based). By [13, Theorem 4.2], $\operatorname{Vol}(G u t s(X)) \geq-V_{8} \chi(G u t s(X))$. Thus, we have $\operatorname{Vol}(M) \geq V_{8}$.

If $\operatorname{Vol}(M)=V_{8}$, then $\operatorname{Vol}(M)=\operatorname{Vol}(G u t s(X))$. We may assume that no complementary region of $M \backslash \backslash X$ is an $I$-bundle, since otherwise we could replace $X$ with a surface which it double covers. By [3, Theorem 5.5], $X$ is totally geodesic, and so $N=M \backslash \backslash X=G u t s(X)$. Regardless of whether $X$ is one- or two-sided, we have $\chi(X)=\frac{1}{2} \chi(\partial N)=\chi(N)=-1$, so $X$ is either a punctured torus, a thricepunctured sphere, or a twice-puncture $\mathbb{R} \mathbb{P}^{2}$. Since $N$ is an acylindrical manifold with $\chi(N)=-1$ and $\operatorname{Vol}(N)=V_{8}$, by Miyamoto's theorem [13. Theorem 4.2], $N$ must have a decomposition into a single regular octahedron of volume $V_{8}$. Thus $M$ decomposes into a single regular octahedron.

Remark. The referee indicated the previous theorem as a natural generalization of our main result and pointed out that there is a sharp example (see [10, Section 6]). The example is m137 from the Snappea census [21], and is obtained by 0-framed surgery on a 2-component link $L$ (see Figure 3 ). To see that m137 contains a closed essential surface, note that there is a thrice-punctured sphere $P$ in the complement of m137 (see the grey surface in the second Figure 31). The surgery along the boundary slope of $P$ gives $S^{2} \times S^{1}$, since the other component of $L$ is unknotted. However, since m137 is hyperbolic, it does not fiber over $S^{1}$ with fiber $P$, and therefore by [8, Theorem 2.0.3], m137 must contain a closed essential surface (which is obtained by carefully tubing together the boundary components of two copies of $P)$.

Theorem 3.6. Suppose that $M$ is an orientable hyperbolic 3-manifold with two cusps. Then $\operatorname{Vol}(M) \geq V_{8}=3.66 \ldots$. If $\operatorname{Vol}(M)=V_{8}$, then $M \cong \mathbb{W}$ or $M \cong \mathbb{W}^{\prime}$.

Proof. By [7, Theorem 3], there is an orientable connected essential separating surface $X_{0} \subset M$ such that $\partial X_{0} \cap\left(T_{2} \cup \cdots \cup T_{n}\right)=\emptyset$, and $\partial X_{0} \cap T_{1}$ is a nonempty union of noncontractible simple closed curves. Moreover, we may assume that there is no compressing annulus of $X_{0}$ along $T_{1}$. By Theorem 3.5. $\operatorname{Vol}(M) \geq V_{8}$. 

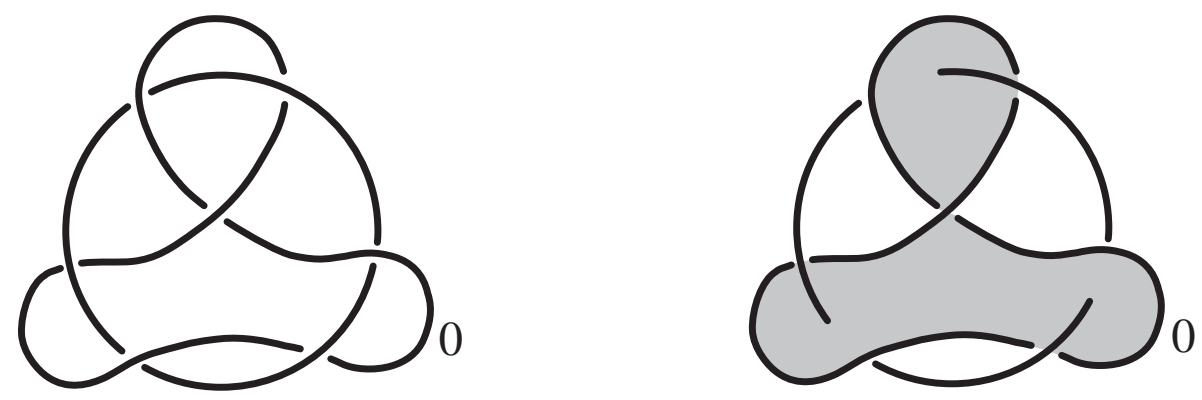

Figure 3. m137 realizes the lower bound in Theorem 3.5 and a thrice-punctured sphere $P$ in $\mathrm{m} 137$.

Now, suppose $\operatorname{Vol}(M)=V_{8}$. By Theorem 3.5, $M$ contains an essential surface $X$ with $\chi(X)=-1$, and the complement of $X$ has a decomposition into a regular octahedron.
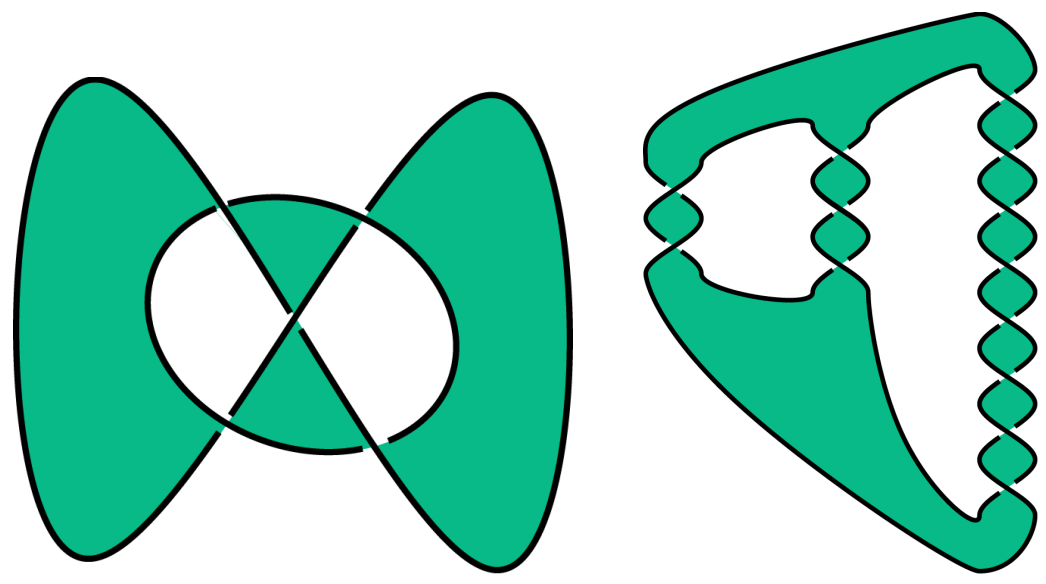

FiguRE 4. A twice-punctured $\mathbb{R}^{2}$ in the Whitehead and $(-2,3,8)$-pretzel link complements

To finish the argument, we take an octahedron $O$ and color the faces alternately black and white. The argument here is somewhat indirect. We know we have two examples, $\mathbb{W}, \mathbb{W}^{\prime}$, and we need to show that there are no more. We glue the black faces of $O$ together in all possible ways to get the possible manifolds $N$. Up to isometry, we get four manifolds $N_{i}, i=1,2,3,4$ with totally geodesic boundary and rank one cusps (see Figure 5, where the black faces being paired are labelled $A$ and $B$ ). Then $\partial N_{1}$ is two pairs of pants, and $\partial N_{i}$ is a single four-punctured sphere, for $i=2,3,4\left(\partial N_{i}\right.$ is shown on the right of Figure 55). Gluing the two boundary components of $N_{1}$ together in a way that gives two cusps gives $\mathbb{W}$ the Whitehead link complement. There are three other manifolds which have boundary a four-punctured sphere. The boundary gets glued to itself by an antipodal map. To get a manifold with two cusps from $N_{i}$, the antipodal map must also identify the rank one cusps of $\partial N_{i}$ which share a common rank one cusp of $N_{i}$. Only $N_{2}$ and $N_{4}$ have boundary which admits an antipodal isometry, so that the quotient 

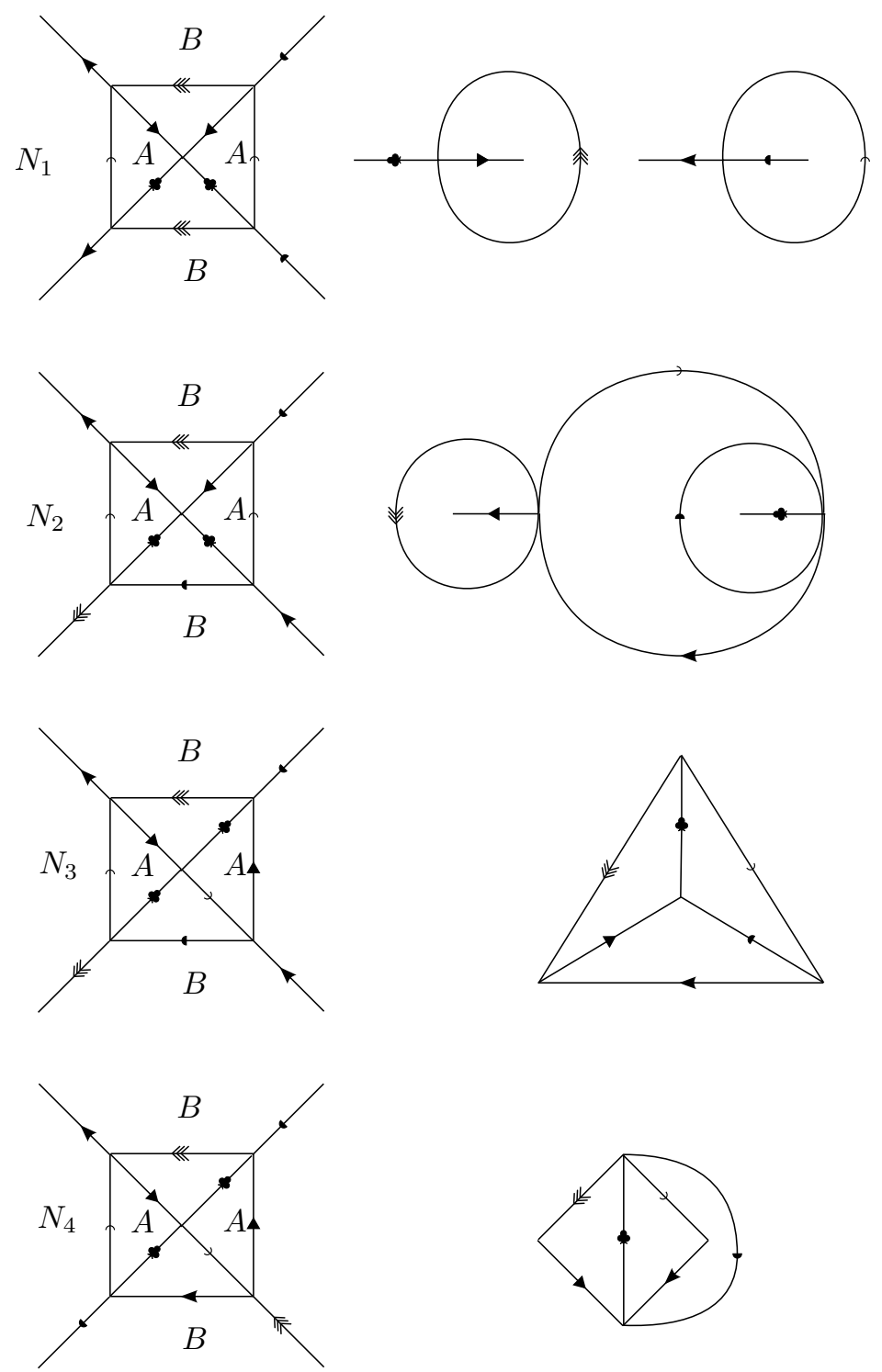

Figure 5. Gluing faces $A$ and $B$ of an octahedron together in pairs to get manifolds with geodesic boundary of volume $V_{8}$

is $X \cong \mathbb{R} \mathbb{P}^{2}-\{x, y\}$. To see that $\partial N_{3}$ cannot have such an antipodal map, notice that $\partial N_{3}$ has isometry group $S_{4}$, for which there is no isometry which acts as an antipodal map. The antipodal isometry preserves the induced triangulation of $\partial N_{i}$ shown on the right of Figure 5 for $i=2,4$. One may check that the quotient by these isometries gives a manifold with two cusps. Any other isometry which had this property must be the same, since the product of two such isometries must fix all four cusps of $\partial N_{i}$, which implies that it is the identity since this is the only holomorphic map of $S^{2}$ fixing four points. One quotient by this map gives back 
$\mathbb{W}$, since $\mathbb{W}$ has a twice-punctured $\mathbb{R}^{2}$ in its complement (see Figure 4 ). The other example must give the $(-2,3,8)$-pretzel link complement $\mathbb{W}^{\prime}$ (in fact, $N_{2}$ corresponds to $\mathbb{W}^{\prime}$ and $N_{4}$ corresponds to $\mathbb{W}$, after one quotients by the unique antipodal map of the boundaries).

\section{Conclusion}

It would be interesting to find the minimal volume orientable hyperbolic manifolds with $n$ cusps. We conjecture that the minimal volume $n$-cusped manifold is realized by a hyperbolic chain link which is minimally "twisted" (see [17] for the definition of chain links) for $n \leq 10$. Rupert Venzke has pointed out to us that for links with $n$ cusps, where $n \geq 11$, the $(n-1)$-fold cyclic cover $\mathbb{W}_{n}$ over one component of $\mathbb{W}$ has volume less than the smallest volume twist link with $n$ cusps, which is a Dehn filling on $\mathbb{W}_{n+1}$. It is probably impossible to use the methods in this paper to determine the smallest volume hyperbolic manifold with $n$ cusps. However, it should be possible to prove the following:

Conjecture 4.1. Let $v_{n}$ be the minimal volume of an n-cusped orientable hyperbolic 3-manifold. Then

$$
\lim _{n \rightarrow \infty} v_{n} / n=V_{8}
$$

As mentioned before, Adams has shown that $v_{n} / n \geq V_{3}$ [1]. It may also be possible to prove:

Conjecture 4.2. The minimal limit volume of nonorientable hyperbolic 3-manifolds is $V_{8}$.

In the Snappea census of 3-manifolds [21, there are four manifolds of volume $V_{8}$ which are nonorientable with a single orientable cusp, which would correspond to the smallest limit volumes of nonorientable manifolds if this conjecture were true. It seems possible that one could prove this conjecture by proving the existence of an essential surface disjoint from the orientable cusp with nontrivial guts.

\section{ACKNOWLEDGEMENTS}

We thank Chris Atkinson and Jeff Weeks for helpful suggestions. We also thank Rupert Venzke and Danny Calegari for a helpful discussion about the minimal volume hyperbolic manifold with $n$ cusps and for correcting a mistaken conjecture in the first version of the paper. We thank the referee for many helpful comments, and for pointing out a strengthening of the main theorem.

\section{REFERENCES}

[1] Colin C. Adams, Volumes of N-cusped hyperbolic 3-manifolds, J. London Math. Soc. (2) 38 (1988), no. 3, 555-565. MR972138 (89k:22020)

[2] Ian Agol, Peter A. Storm, and William P. Thurston, Lower bounds on volumes of hyperbolic Haken 3-manifolds, J. Amer. Math. Soc. 20 (2007), no. 4, 1053-1077 (electronic), arXiv:math.DG/0506338, with an appendix by Nathan Dunfield. MR 2328715 (2008i:53086)

[3] Danny Calegari, Michael H. Freedman, and Kevin Walker, Positivity of the universal pairing in 3 dimensions, J. Amer. Math. Soc. 23 (2010), no. 1, 107-188. MR2552250

[4] Richard D. Canary and Darryl McCullough, Homotopy equivalences of 3-manifolds and deformation theory of Kleinian groups, Mem. Amer. Math. Soc. 172 (2004), no. 812, xii+218. MR2096234 (2005j:57027)

[5] Chun Cao and G. Robert Meyerhoff, The orientable cusped hyperbolic 3-manifolds of minimum volume, Invent. Math. 146 (2001), no. 3, 451-478. MR.1869847 (2002i:57016) 
[6] Huai-Dong Cao and Xi-Ping Zhu, A complete proof of the Poincaré and geometrization conjectures - application of the Hamilton-Perelman theory of the Ricci flow, Asian J. Math. 10 (2006), no. 2, 165-492, arXiv:math/0612069v1. MR2233789 (2008d:53090)

[7] M. Culler and P. B. Shalen, Bounded, separating, incompressible surfaces in knot manifolds, Invent. Math. 75 (1984), no. 3, 537-545. MR735339 (85k:57010)

[8] Marc Culler, C. McA. Gordon, J. Luecke, and Peter B. Shalen, Dehn surgery on knots, Ann. of Math. (2) 125 (1987), no. 2, 237-300. MR881270 (88a:57026)

[9] Marc Culler and Peter B. Shalen, Volumes of hyperbolic Haken manifolds. I, Inventiones Mathematicae 118 (1994), no. 2, 285-329. MR1292114 (95g:57023)

[10] Nathan M. Dunfield, Examples of non-trivial roots of unity at ideal points of hyperbolic 3-manifolds, Topology 38 (1999), no. 2, 457-465. MR1660313 (2000b:57021)

[11] David Gabai, Robert Meyerhoff, and Peter Milley, Minimum volume cusped hyperbolic threemanifolds, J. Amer. Math. Soc. 22 (2009), no. 4, 1157-1215, arXiv:0705.4325. MR2525782

[12] Bruce Kleiner and John Lott, Notes on Perelman's papers, Geom. Topol. 12 (2008), no. 5, 2587-2855, arXiv:math.DG/0605667. MR2460872

[13] Yosuke Miyamoto, Volumes of hyperbolic manifolds with geodesic boundary, Topology 33 (1994), no. 4, 613-629. MR.1293303 (95h:57014)

[14] John Morgan and Gang Tian, Ricci flow and the Poincaré conjecture, Clay Mathematics Monographs, vol. 3, American Mathematical Society, Providence, RI, 2007, arXiv:math/0607607v2. MR2334563 (2008d:57020)

[15] John Morgan and Gang Tian, Completion of the proof of the geometrization conjecture, preprint, 2008, arXiv.org:0809.4040.

[16] John W. Morgan, On Thurston's uniformization theorem for three-dimensional manifolds, The Smith conjecture (New York, 1979), Academic Press, Orlando, FL, 1984, pp. 37-125. MR:758464

[17] Walter D. Neumann and Alan W. Reid, Arithmetic of hyperbolic manifolds, Topology '90 (Columbus, OH, 1990), Ohio State Univ. Math. Res. Inst. Publ., vol. 1, de Gruyter, Berlin, 1992, pp. 273-310. MR1184416 (94c:57024)

[18] Grisha Perelman, Ricci flow with surgery on three-manifolds, arXiv:math.DG/0303109

[19] - The entropy formula for the Ricci flow and its geometric applications, arXiv:math.DG/0211159

[20] John G. Ratcliffe, Foundations of hyperbolic manifolds, second ed., Graduate Texts in Mathematics, vol. 149, Springer, New York, 2006. MR2249478 (2007d:57029)

[21] Jeffery Weeks, SnapPea: A computer program for creating and studying hyperbolic 3manifolds, www.geometrygames.org.

[22] Han Yoshida, Volumes of orientable 2-cusped hyperbolic 3-manifolds, Kobe J. Math. 18 (2001), no. 2, 147-161. MR.1907670 (2003f:57036)

Department of Mathematics, University of California, Berkeley, 970 Evans Hall \#3840, Berkeley, California 94720-3840

E-mail address: ianagol@math.berkeley.edu 